\title{
Effects of Helicobacter pylori infection and interleukin 6 on the expression of ITIH4 in human gastric cancer cells
}

\author{
Dadao Jing, Jie Jin, Zhu Mei, Qingqing Zhu, Yingying Lu, Xingpeng Wang \\ Department of Gastroenterology, Shanghai General Hospital, Shanghai Jiao Tong University School of Medicine, Shanghai, China \\ Contributions: (I) Conception and design: D Jing, X Wang; (II) Administrative Support: D Jing; (III) Provision of study materials or patients: J Jin, Z \\ Mei; (IV) Collection and assembly of data: Q Zhu, Y Lu; (V) Data analysis and interpretation: D Jing, Z Mei, X Wang; (VI) Manuscript writing: all \\ authors; (VII) Final approval of manuscript: All authors. \\ Correspondence to: Dadao Jing; Xingpeng Wang. Department of Gastroenterology, Shanghai General Hospital, Shanghai Jiao Tong University School \\ of Medicine, 650 Xinsongjiang Rd, Songjiang, Shanghai, China. Email: dadaojing@126.com; richardwangxp@163.com.
}

\begin{abstract}
Background: Human inter- $\alpha$-trypsin inhibitor heavy chain 4 (ITIH4) is an acute phase response protein that is positively regulated by interleukin-6 (IL-6), suggesting that ITIH4 can be used as an early serological biomarker of gastric cancer.
\end{abstract}

Methods: The mechanism of ITIH4 elevation in gastric cancer cells was investigated via Helicobacter pylori (H. pylori) with or without recombinant human interleukin-6 (rhIL-6) in human gastric cell line AGS cells. The messenger RNA (mRNA) expressions of ITIH4 and IL-6 in AGS cells were detected with real-time quantitative polymerase chain reaction (qPCR), while the expression of ITIH4 protein and IL-6 in AGS cells was detected with Western blot and enzyme-linked immunosorbent assay (ELISA) methods.

Results: We found that the mRNA and protein expressions of ITIH4 started to increase synchronously at 3 hours (h), peaked at $12 \mathrm{~h}$, and then declined to normal a level at $24 \mathrm{~h}$ in AGS cells after H. pylori infection. Interestingly, the expression patterns of ITIH4 were positively correlated to IL-6 expression levels. In the ITIH4 expression experiment with IL-6 administration, the expression level of ITIH4 began to increase at a concentration of $6.25 \mathrm{ng} / \mathrm{mL}$ with rhIL-6 administration, reach a peak, and plateau at a concentration of $25 \mathrm{ng} / \mathrm{mL}$ of rhIL-6. The expression patterns of ITIH4 in AGS cells induced by $H$. pylori infection were closely related to the infection of $H$. pylori and the level of IL-6.

Conclusions: The detection of ITIH4 and IL-6 during H. pylori infection may be useful for the screening of early gastric cancer.

Keywords: Gastric cancer; Helicobacter pylori (H. pylori); human inter- $\alpha$-trypsin inhibitor heavy chain 4 (ITIH4); interleukin-6 (IL-6)

Submitted Apr 05, 2020. Accepted for publication Jul 10, 2020.

doi: $10.21037 /$ tcr-20-1766

View this article at: http://dx.doi.org/10.21037/tcr-20-1766

\section{Introduction}

Human inter- $\alpha$-trypsin inhibitor heavy chain 4 (ITIH4) is a $120 \mathrm{kDa}$ serum glycoprotein mainly secreted by the liver and is extensively involved in liver development and regeneration $(1,2)$. One study showed that ITIH4 circulates in the blood at a concentration of about $100 \mathrm{ng} / \mathrm{mL}$ (3). It is an acute phase responsive protein and is positively regulated by interleukin 6 (IL-6) $(4,5)$. Previous studies have shown that the expression of ITIH4 in cancer patients is related to tumorigenesis and cancer progression, suggesting that ITIH4 may be used as a serological marker for diagnosis cancer (6-8). Our cytological studies have also shown that $70 \mathrm{kDa}$ ITIH4 protein fragments are expressed in gastric cancer cells. In addition, our study also found that elevated serum ITIH4 in patients with early gastric cancer was strongly associated with Helicobacter pylori $(H$. pylori) infection and serum IL-6 levels. So far, however, 
the effects of ITIH4 on the occurrence and development of gastric cancer, and the mechanism of its elevation in early gastric cancer serum, remain unclear. A large number of studies have demonstrated that $H$. pylori infection can cause chronic inflammation, atrophy, intestinalization, dysplasia and metaplasia of gastric mucosal epithelial cells, and canceration $(9,10)$. Other studies have shown that IL-6 receptor expression is closely related to the emergence and development of gastric cancer (11).

To evaluate the exact functions of IHIT4 expression in gastric cancer, we treated human AGS gastric cancer cells with recombinant human interleukin 6 (rhIL-6) and $H$. pylori, and observed the effects of IL-6 and $H$. pylori on ITIH4 expression in gastric cancer cells. The results yielded from this experiment elucidated the mechanism of ITIH4 elevation in early gastric cancer serum and could be valuable in further clarifying the possibility of ITIH4 as a serological marker of early gastric cancer.

\section{Methods}

\section{Cell culture}

Human gastric cancer cell line AGS (Shanghai institute of cell research, Chinese academy of sciences) was selected and cultured in RPMI 1640 medium (Gibco, USA) containing 10\% newborn bovine serum (Hyclone, USA), 100 unit $(\mathrm{U}) / \mathrm{mL}$ penicillin, and $100 \mathrm{U} / \mathrm{mL}$ streptomycin, and placed in $5 \% \mathrm{CO}_{2}$ cell incubator at $37{ }^{\circ} \mathrm{C}$. When the cells adhered to the $80-90 \%$ confluence, they were digested and subcultured with $0.25 \%$ trypsin solution detachment. This study was approved by ethical committee of Shanghai General Hospital (Approval\#: 2017KY210) and complied with the Declaration of Helsinki (As revised in 2013). Informed consent was not required because we only used human cancer cell line in the entire experiment.

\section{H. pylori strain culturing}

H. pylori bacteria were resuscitated and inoculated into brain heart infusion containing $7 \%$ defibrinated sheep blood, 0.4\% IsoVitaleXEnrichment (Becton, Dickinson, and Co., Franklin Lakes, NJ, USA), 0.08\% amphotericin $\mathrm{B}, 0.2 \%$ vancomycin, and $0.5 \%$ sulfa synergist infusion agar medium (USA, Becton, Dickinson, and Co., Franklin Lakes, NJ, USA). This was cultured at $37{ }^{\circ} \mathrm{C}$ in a three-gas incubator containing $5 \% \mathrm{O}_{2}, 10 \% \mathrm{CO}_{2}, 85 \% \mathrm{~N}_{2}$, and $95 \%$ relative humidity. After 3-5 days, the typical colonies were needle-point-like (diameter 1-2 days, the typical colonies were needle-point-like (diameter 1nergihad increased, the colonies fused on the surface of the culture medium to form a translucent bacterial moss. The motor status of $H$. pylori was observed under a dark-field microscope. The morphological identification of $H$. pylori strains was verified by Gram staining, Giemsa staining, and WarthinStarrysilver staining.

\section{Co-culture of $H$. pylori and AGS cells}

To evaluate IHIT4 gene and protein expression in $H$. pylori-infected AGS cells, we performed the following steps for AGS cells culture. First, AGS cells were taken in logarithmic growth phase, routinely digested with sterile $0.25 \%$ trypsin, and were counted. Cells were adjusted with antibiotic-free RPMI 1640 medium with $10 \%$ fetal bovine serum (FBS) at 5 bov $^{5}$ cells density $/ \mathrm{ml}$ and inoculated into a 12-well cell culture plate at $200 \mu \mathrm{L}$ per well. Then, each well was supplemented with $0.8 \mathrm{~mL}$ RPMI 1640 culture medium with $10 \% \mathrm{FBS}$, and the final volume of each well was $1 \mathrm{ml}$. These cells were placed in a $5 \% \mathrm{CO}_{2}$ incubator at $37^{\circ} \mathrm{C}$ to culture for 24 hours (h). Second, $4-5$ colonies with a diameter of about $1 \mathrm{~mm}$ that had been cultured for 5-7 days on the brain heart infusion agar medium with an inoculating loop and saline were sterilized with $3-5 \mathrm{~mL}$ of antibiotic-free 10\% FBS RPMll640 medium; this adjusted the bacterial concentration to $1 \times 10^{8}$ colony-forming units $(\mathrm{CFU}) / \mathrm{mL}$. Third, the cell culture plate was taken out for $2 \mathrm{~h}$, the culture supernatant was removed, and then divided into five groups: A, B, C, D, and $\mathrm{E}$ with 6 duplicate wells in each group. Separately, groups A, B, C, D, and E were combined with $100 \mathrm{~L}$ of bacterial solution, AGS cells, and H. pylori that were incubated at a ratio of 1:100 multiplicity of infection (MOI). The AGS cells served as the control group. Then, $1 \mathrm{ml}$ of fresh, serum-free, antibiotic-free RPMI1640 culture solution was added into each well and cultured in a $5 \% \mathrm{CO}_{2}$ incubator at $37{ }^{\circ} \mathrm{C}$ for $1,3,6,12$, and $24 \mathrm{~h}$ for groups A, B, C, D, and E, respectively. The culture conditions are described in Table 1. Cells were harvested for ITIH4 gene and protein analysis according to the set time.

\section{rbIL-6-induced ITIH4 expression}

To assess the effects of IL-6 on ITIH4 gene expression in AGS cells, we harvested AGS cells in logarithmic growth phase with sterile $0.25 \%$ trypsin digestion. Then, cells were counted and cell density was adjusted at $5 \times 10^{5}$ cells $/ \mathrm{mL}$ 
Table 1 Co-culture of H. pylori and AGS cell lines

\begin{tabular}{lcccccc}
\hline Variable & A & B & C & D & E & Control \\
\hline AGS cell & $1 \times 10^{5}$ & $1 \times 10^{5}$ & $1 \times 10^{5}$ & $1 \times 10^{5}$ & $1 \times 10^{5}$ & $1 \times 10^{5}$ \\
H. pylori $(\mathrm{CFU} / \mathrm{mL})$ & $1 \times 10^{7}$ & $1 \times 10^{7}$ & $1 \times 10^{7}$ & $1 \times 10^{7}$ & $1 \times 10^{7}$ & 1.0 \\
Total volume $(\mathrm{mL})$ & 1.0 & 1.0 & 1.0 & 1.0 & 12 & 24 \\
Time of culture (hour) & 1 & 3 & 6 & 1.0 & \\
\hline
\end{tabular}

H. pylori, Helicobacter pylori; CFU, colony-forming-unit.

Table 2 Recombinant human interleukin 6 induces ITIH4 expression

\begin{tabular}{|c|c|c|c|c|c|}
\hline Variable & $A$ & $\mathrm{~B}$ & $\mathrm{C}$ & $\mathrm{D}$ & E \\
\hline rhlL-6 (ng/mL) & 0 & 6.25 & 12.5 & 25.0 & 50.0 \\
\hline Total volume (mL) & 1.0 & 1.0 & 1.0 & 1.0 & 1.0 \\
\hline Time of culture (hour) & 24 & 24 & 24 & 24 & 24 \\
\hline
\end{tabular}

rhIL-6, recombinant human interleukin 6; ITIH4, inter- $\alpha$-trypsin inhibitor heavy chain 4.

with antibiotic-free 10\% FBS RPMI 1640 medium. The cells were inoculated into a 24-well cell culture plate, 200 in AGS cells, we harvested AGS cells in logarithmic growth phase cultured in RPMI 1640 medium (Gibco, USA) which was then incubated in a $5 \% \mathrm{CO}_{2}$ incubator at $37{ }^{\circ} \mathrm{C}$ for $24 \mathrm{~h}$. Previous cells were treated with 6.25, 12.5, 25.5, and $50.0 \mathrm{ng} / \mathrm{mL}$ rhIL-6 (PeproTech EC, UK) and antibiotic-free RPMI 1640 cell culture medium, respectively (Table 2). After the cells were incubated for $24 \mathrm{~h}$ at $37^{\circ} \mathrm{C}, 5 \% \mathrm{CO}_{2}$ incubator and were digested, the cells with $0.25 \%$ sterile trypsin, RNA and protein were extracted and stored in $-70{ }^{\circ} \mathrm{C}$ low temperature refrigerator for later use.

\section{Determination of IL-6 and ITIH4 mRNA expression in cells by real-time quantitative polymerase chain reaction (qPCR)}

\section{qPCR}

Total RNA was isolated from AGS cells with $H$. pylori and IL-6 administration using TRIzol and was then used for complementary DNA synthesis using random primers. Reverse transcriptasequantitative polymerase chain reaction (PCR) was performed following standard procedures. PCR conditions for ITIH4 were 35 cycles of $95{ }^{\circ} \mathrm{C}$ for 15 seconds (s); $58{ }^{\circ} \mathrm{C}$ for 15 seconds; and $72{ }^{\circ} \mathrm{C}$ for 10 seconds, conditions for IL-6 were 33 cycles of $95{ }^{\circ} \mathrm{C}$ for 30 seconds; $56{ }^{\circ} \mathrm{C}$ for 30 seconds; and $72{ }^{\circ} \mathrm{C}$ for 30 seconds, and conditions for the house keeping gene
GAPDH were 24 cycles of $95{ }^{\circ} \mathrm{C}$ for 30 seconds; $58^{\circ} \mathrm{C}$ for 30 seconds; and $72{ }^{\circ} \mathrm{C}$ for 30 seconds. The primer pairs of the expected products are displayed in Table 3. For each specimen in duplicate wells, a sample without cDNA was set as a negative control, and a blank buffer without cDNA and no probe was used as a blank control. The ABI7900 fluorescence quantitative PCR instrument collected the genes to be tested, and the internal reference GAPDH was used to amplify the fluorescence signals of each cycle, using SDS2.0 software (Applied Biosystems, USA) for fluorescence collection and data analysis. The detection mode was absolute quantification. Sequence Detector 1.7 software analysed the cycle threshold $(\mathrm{Ct})$ value and $\mathrm{Ct}$ threshold value (threshold value $=$ standard deviation of baseline signal $\times 10$ ). The $c D N A t$ value reflects the number of reaction cycles required when the template is amplified to a certain number of copies (in an exponentially rising period): the larger the $\mathrm{Ct}$ value, the smaller the amount of starting template participating in the reaction, and vice versa. Additional calculation included $\Delta \Delta \mathrm{Ct}$ and relative quantity (RQ) value. This value was arrived at by calculating the average $\mathrm{Ct}$ value of each specimen, and taking the target gene $\mathrm{Ct}$ value minus GAPDH Ct value as the $\mathrm{d} t$ value as follows: $\Delta \Delta \mathrm{Ct}=\Delta \mathrm{Ct}$ (experimental group) $-\Delta \mathrm{Ct}$ (control group); RQ $=2^{-\Delta \Delta \mathrm{Ct}}$. Amplified products were resolved by $2-3 \%$ agarose gel electrophoresis and $100 \mathrm{~V}$ electrophoresis for $45 \mathrm{~min}$; the gel was observed under UV light, and a photo of the bottom was taken. 
Table 3 Polymerase chain reaction primer sequence of this experiment

\begin{tabular}{|c|c|c|c|}
\hline Objects & Primer sequences & $\begin{array}{c}\text { Amplified } \\
\text { fragment (bp) }\end{array}$ & $\begin{array}{c}\text { Annealing } \\
\text { temperature }\left({ }^{\circ} \mathrm{C}\right)\end{array}$ \\
\hline ITIH4 & Forward 5'-GGTCTCATCCCGATTTGC-3', reverse 5'-TGATCCCTGGGTAGGTCAT-3' & 155 & 58 \\
\hline GAPDH & Forward 5'-AACGGATTTGGTCGTATTG-3', reverse 5'-GGAAGATGGTGATGGGATT-3' & 208 & 58 \\
\hline
\end{tabular}

ITIH4, inter- $\alpha$-trypsin inhibitor heavy chain 4; IL-6, interleukin-6; GAPDH, glyceraldehyde 3-phosphate dehydrogenase.

\section{Western blot method to detect the expression of ITIH4 and IL-6 protein in gastric cancer cells}

Cell lysates were prepared, and protein concentration measured by bicinchoninic acid (BCA) protein assay reagent. An equal amount of protein $(20 \mathrm{ng})$ were loaded on a $6 \%$ or $10 \%$ SDS-polyacrylamide gel and transferred to a polyvinylidene fluoride (PVDF) membrane. The transferred protein was blocked in $5 \%$ fat-free dry milk in phosphate buffered saline (PBS) containing $0.1 \%$ Tween-20 (PBST) for $1 \mathrm{~h}$ at room temperature. Then, the membrane was incubated with a primary antibody (rabbit anti-human ITIH4 antibody, Abcam, Cat\#ab180139, USA; rabbit anti-human IL-6 antibody, Abcam, Cat\# 1457-1, RRID:AB_562150, USA) in 5\% skimmed milk powder in $3 \% \mathrm{PBS}$ at $4{ }^{\circ} \mathrm{C}$ overnight and incubated with the respective horseradish peroxidase (HRP)-conjugated secondary antibodies (goat anti-rabbit IgG-HRP, Cat\#G-21234 Invitrogen, USA) at 1:2,000 for $2 \mathrm{~h}$ and washed again with PBST. An enhanced chemiluminescence (ECL) detection kit (Shanghai Shengsheng Biotechnology Co., Ltd.) was used to visualize the expressed proteins. The Gene Genius imaging system was used for scanning and imaging, and each procedure was repeated at least three times.

\section{Expression levels of ITIH4 and IL-6 in cell lysate}

ITIH4 and IL-6 levels in cell lysate were measured by enzyme-linked immunosorbent assay (ELISA) according to the manufacturer's protocol (Wuhan Huamei Biological Engineering Co., Ltd. and R \& D).

\section{Image analysis}

Gel-Pro image analysis software was used to analyse the Western blot results. The detection results were described in terms of relative absorbance. The relative absorbance was the ratio of the grey level of the target band to the grey level of the internal reference GAPDH band.

\section{Statistical analysis}

The obtained data are expressed as mean $\pm \mathrm{SD}$. The relevant data were analysed by SPSS 24.0 statistical software. The data between the two groups were analyzed by paired $t$-test. The data between multiple groups were analyzed by a single factor analysis of variance. A P value $<0.05$ was considered statistically significant.

\section{Results}

ITIH4 mRNA expression of AGS cells induced by H. pylori infection (amplification curve of ITIH4 gene by real-time qPCR)

After harvesting $H$. pylori-infected AGS cells at the set time, RNA was isolated and extracted, and real-time quantitative PCR was performed to analyze the differential expression of ITIH4 mRNA in AGS cells. The phase of the fluorescence intensity detected by the PCR instrument was uniformly set to the annealing period of the amplification reaction. In this experiment, ITIH4 and gene amplification were performed at $60{ }^{\circ} \mathrm{C}$ with 30 seconds as the annealing period. After 35 cycles of amplification reactions had elapsed, the system analyzed the fluorescence intensity growth index (DRn) of each reaction tube collected during each cycle of reaction and drew the amplification curve of each reaction tube (Figure 1A,B,C). The amplification curve of each sample had reached the plateau stage. In contrast, none of the melting curves detected primer dimers and other non-specific fluorescent signals (Figure 1D,E,F).

\section{Differential expression of ITIH4 $m R N A$ in AGS cells with H. pylori infection}

The expression level of ITIH4 mRNA in AGS cells began to increase $3 \mathrm{~h}$ after $H$. pylori infection, reached a peak at $6 \mathrm{~h}$, and decreased to maintain low levels at $24 \mathrm{~h}$. The statistics of ITIH4 in three independent experiments showed that the 
A

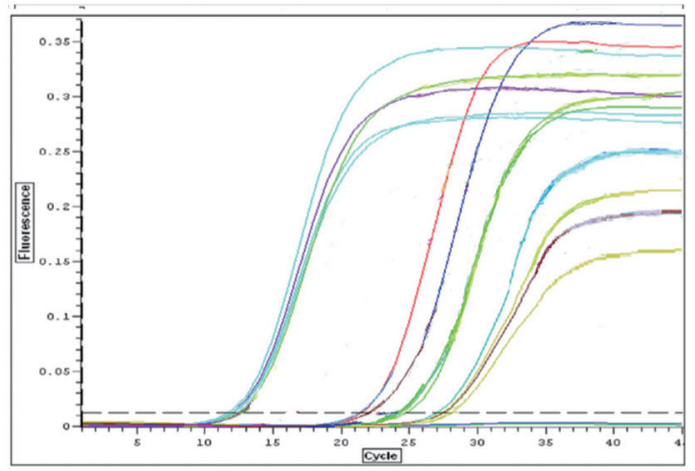

B

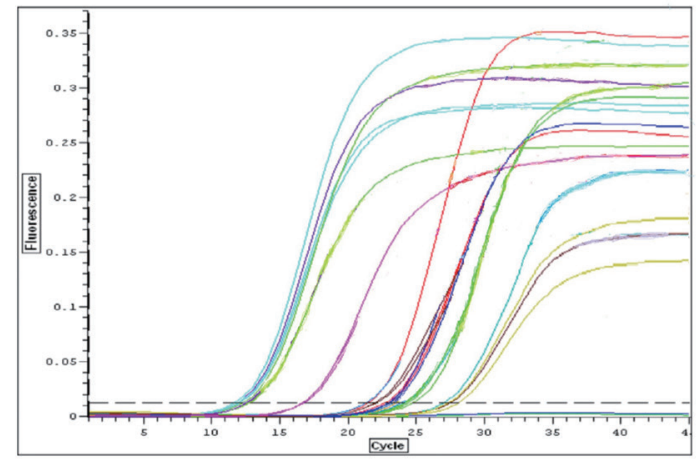

C

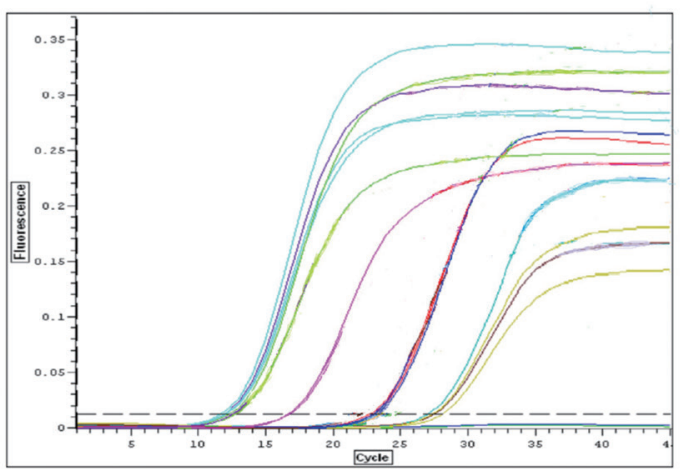

D

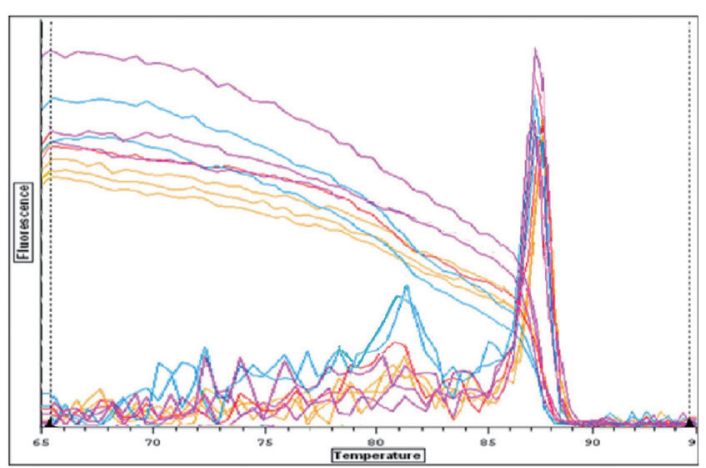

$\mathrm{E}$

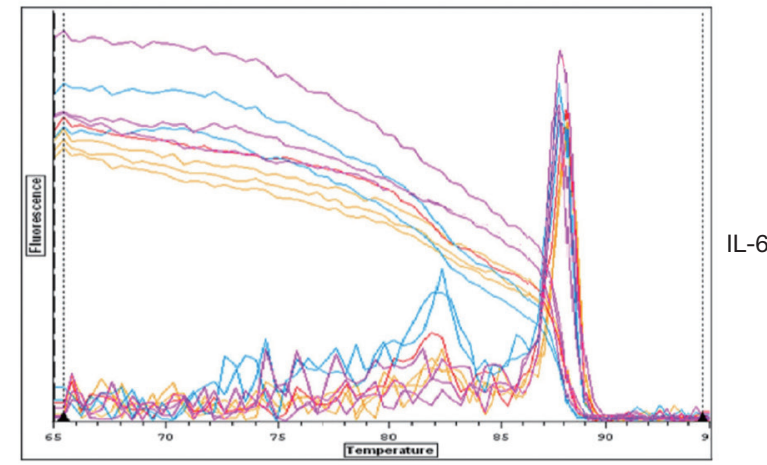

F

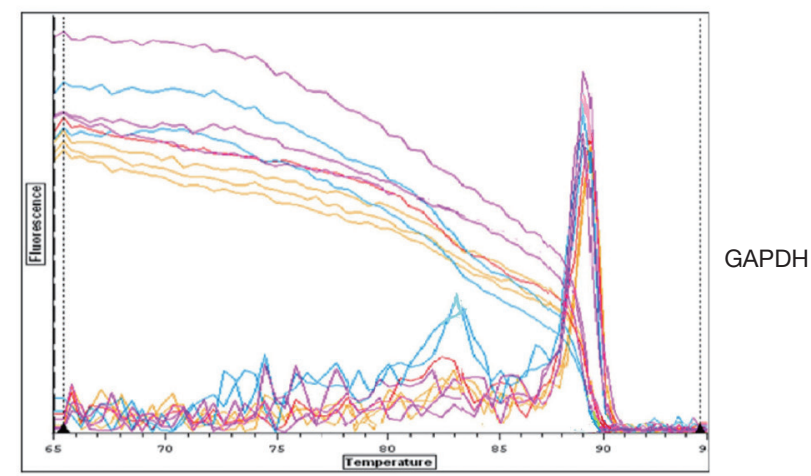

Figure 1 The dynamic and melting curve of the ITIH4 and IL-6 genes by real-time PCR. (A, B, and C) are the amplification curves of ITIH4, IL-6, and GAPDH. (D, E, and F) are the melting curve of ITIH4, IL-6, and GAPDH, respectively. PCR, polymerase chain reaction; ITIH4, inter- $\alpha$-trypsin inhibitor heavy chain 4; IL-6, interleukin-6.

expression of ITIH4 in AGS cells was highest at $6 \mathrm{~h}$ after $H$. pylori infection $(\mathrm{P}<0.01)$ (Figure $2 A, B)$.

\section{Differential expression of IL-6 $m R N A$ in AGS gastric cancer cells after $H$. pylori infection}

To further clarify the mechanism of enhanced ITIH4 mRNA expression in AGS cells after $H$. pylori infection, we also analyzed the expression of IL-6 in $H$. pylori-infected gastric cancer cells. The results are shown in Figure 2B,C. The expression level of IL-6 mRNA in AGS cells began to increase $3 \mathrm{~h}$ after $H$. pylori infection, and reached a peak at $6 \mathrm{~h} \mathrm{IL-6}$ values in three independent experiments showed that the expression of IL-6 mRNA in AGS cells increased at the same time as ITIH4, reached a peak at $6 \mathrm{~h}$, and decreased after $12 \mathrm{~h}$, but remained at a high level $(\mathrm{P}<0.05)$ (Figure 2B,C). We also performed correlation analysis of ITIH4 and IL-6 expression. The results revealed that the change of ITIH4 expression level in AGS cells was significantly correlated with the increase of IL- 6 expression level $(\mathrm{r}=0.897, \mathrm{P}<0.01)$ (Figure 2D). These data indicated that $H$. pylori could induce the expressions of ITIH4 and IL- 6 genes in a time 
A
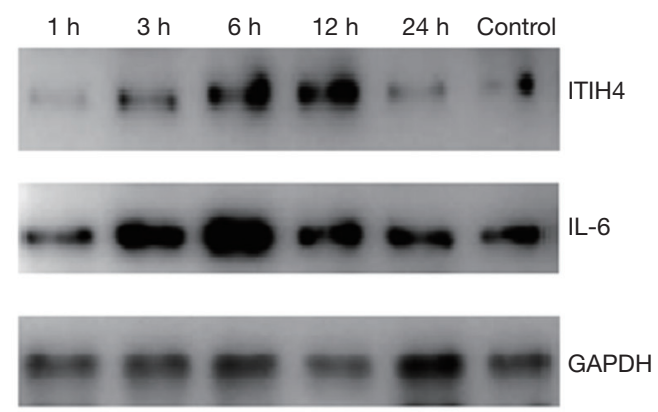

C

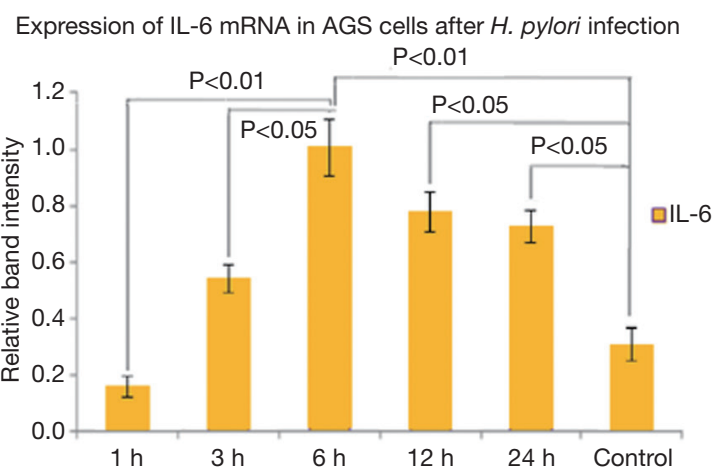

B

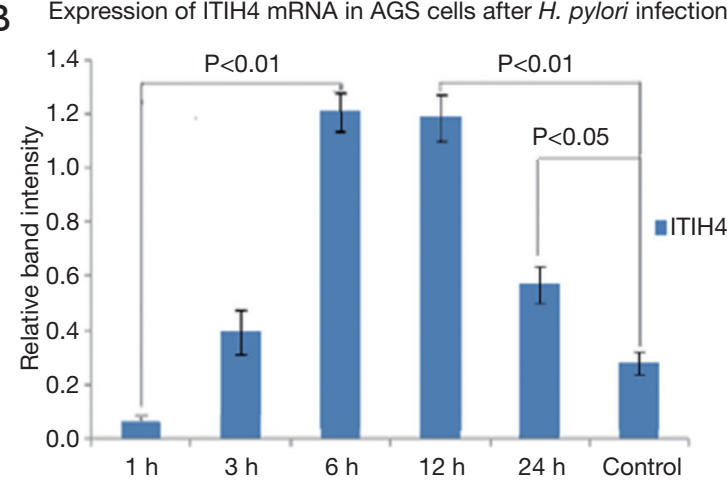

D

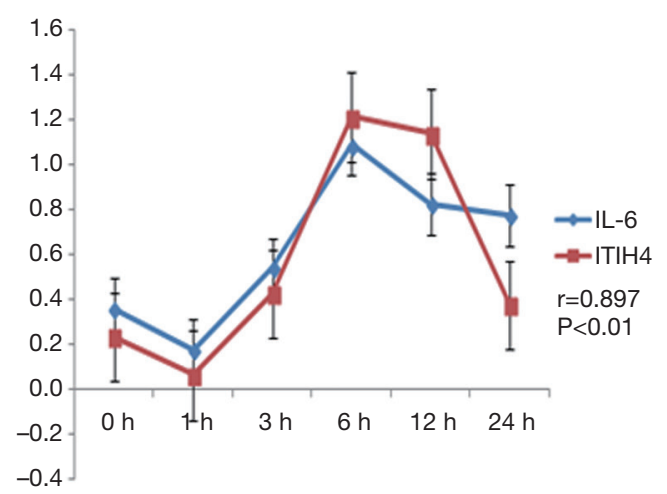

Figure 2 Expressions of ITIH4 and IL-6 genes and proteins in AGS gastric cancer cells after H. pylori infection. (A) is the qPCR product of ITIH4 and IL-6 genes in AGS cells with $H$. pylori infection by agarose gel at different time points. (B) is the comparison of ITIH4 gene expression quantity in AGS gastric cancer cells after $\mathrm{H}$. pylori infection. (C) is the expression of IL-6 mRNA in AGS gastric cancer cells after H. pylori infection. (D) is the correlation analysis of ITL-6 and ITIH4 mRNA changes in AGS cells after H. pylori infection. ITIH4, inter- $\alpha$ trypsin inhibitor heavy chain 4; IL-6, interleukin-6; qPCR, quantity polymerase chain reaction; H. Pylori, Helicobacter pylori.

dependent manner. Moreover, the expression pattern of ITIH4 showed a strong positive correlation with IL6expression.

\section{Changes of ITIH4 and IL-6 protein expression in gastric cancer cells after $\mathrm{H}$. pylori infection}

To further clarify the relationship between ITIH4 expression and $H$. pylori infection and IL-6 expression, Western blot and ELISA techniques were used to detect the expression levels of ITIH4 and IL-6 proteins in lysates of AGS cells infected with $H$. pylori. AGS cells were collected at a predetermined time to obtain the cell lysates. Western blot analysis showed that ITIH4 protein increased $3 \mathrm{~h}$ after $H$. pylori infection and reached a peak at $12 \mathrm{~h}$. In contrast, the IL- 6 protein also started to increase at $3 \mathrm{~h}$ after infection, but reached its peak at $6 \mathrm{~h}$ (Figure 3A). ELISA results revealed that the expression of ITIH4 and IL-6 proteins was similar to the Western blot pattern, but the level of IL-6 was higher than that of ITIH4 at several time points (Figure 3B). This analysis confirmed the expression pattern of ITIH4 and IL-6 in AGS cells with $H$. pylori infection.

\section{Effect of recombinant buman interleukin 6 (rbIL-6) on the expression of ITIH4 in gastric cancer cells}

Different concentrations of rhIL-6 were used to induce AGS gastric cancer cells. Real-time quantitative PCR showed that as the concentration of rhIL-6 increased, the expression of ITIH 4 mRNA increased in a dose-dependent manner (Figure 4A). ELISA also showed that the level of ITIH4 protein in the AGS cell lysate increased linearly as the concentration of rhIL-6 increased. At a concentration 

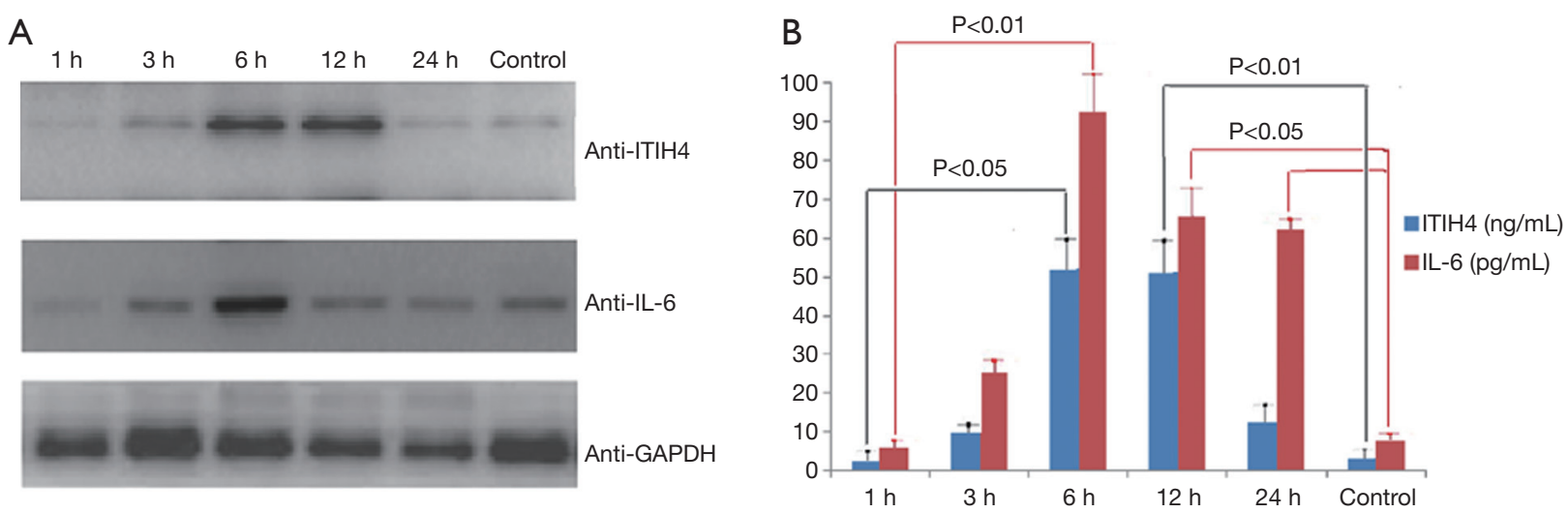

Figure 3 Expression of IL-6 and ITIH4 in AGS gastric cancer cells after H. pylori infection. (A) is ITIH4 and IL-6 expression in AGS cell by Western blot. (B) is the quantitative comparison of ITIH4 and IL-6 protein in AGS cells after H. Pylori infection by ELISA. ITIH4, inter- $\alpha$-trypsin inhibitor heavy chain 4; IL-6, interleukin-6; H. Pylori, Helicobacter pylori; ELISA, enzyme-linked immunosorbent assay.
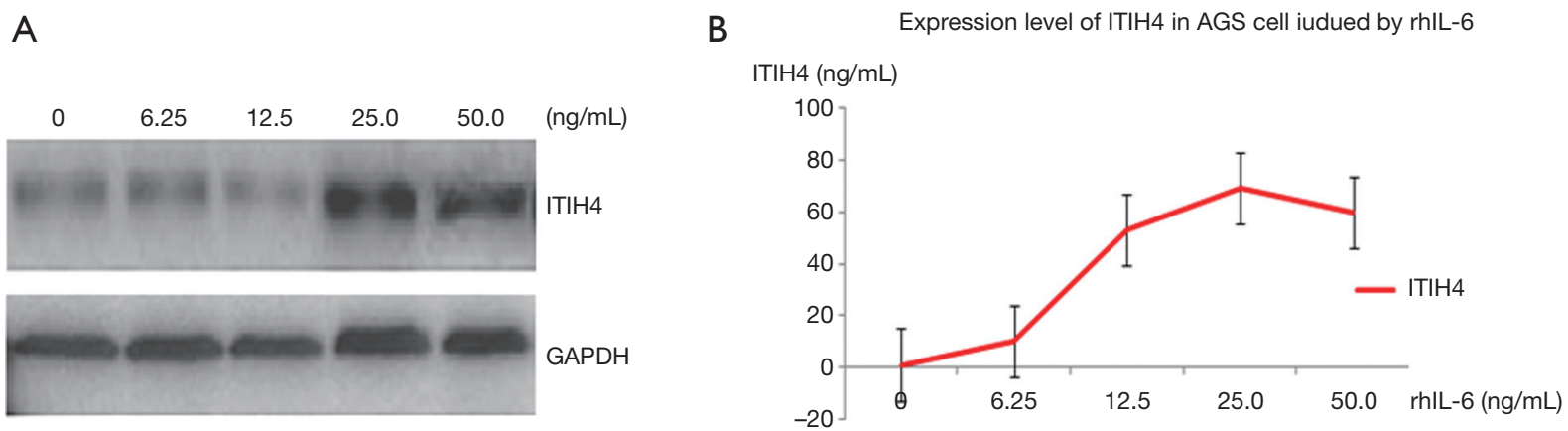

Figure 4 Expression of ITIH4 mRNA in AGS cells induced by rhIL-6. (A) is the ITIH4 protein expression in AGS cells with different amounts of IL-6 by Western blot. (B) is the expression level of ITIH4 in AGS cell lysate induced with rhIL-6 by ELISA. ITIH4, inter$\alpha$-trypsin inhibitor heavy chain 4; rhIL-6, recombinant human interleukin-6; H. Pylori, Helicobacter pylori; ELISA, enzyme-linked immunosorbent assay.

of $50 \mathrm{ng} / \mathrm{mL}$, the expression of ITIH4 reached a plateau (Figure 4B). This suggests that the expression of ITIH4 in gastric cancer cells is closely related to the level of IL-6.

\section{Discussion}

The course of gastric cancer is a multifactor and multistep process. The occurrence and development of gastric cancer generally progresses through chronic superficial gastritis, atrophy, intestinalization, dysplasia, metamorphosis, and finally results in gastric mucosal cancer (9). More than $60 \%$ of gastric cancer cases originate from H. pylori (12), with smoking and eating particular foods, like pickled vegetables, also frequently being linked to this cancer (13). After its initial discovery, ample research has confirmed
H. pylori as a class I carcinogen of gastric cancer $(14,15)$. Chronic inflammation, ulcers, atrophy, intestinal dysplasia, and dysplasia of gastric mucosa are caused by chronic $H$. pylori infection, with many cytokines and inflammatory factors being involved in this process $(9,10)$. Recent studies have shown that peripheral serum levels of IL-6, IL-1, transforming growth factor $\beta$ (TGF- $\beta$ ), and tumor necrosis factor $\alpha$ (TNF- $\alpha$ ) are significantly increased in patients with gastric cancer patients $(5,11,16)$. Some studies have suggested that the increase of serum IL-6 and IL$6 \mathrm{R}$ is closely related to the prognosis of tumors $(16,17)$. Therefore, it is believed that serum IL-6 level can be used as a marker for the serological diagnosis and prognosis of gastric cancer.

Previous studies have shown that serum ITIH4 level is 
specifically elevated in patients with early gastric cancer $(18,19)$. In this study, H. Pylori was first co-cultured with AGS cells. The results showed that the expression levels of ITIH4 and IL-6 genes in AGS cells increased simultaneously $3 \mathrm{~h}$ after $H$. pylori infection and reached a peak at $6 \mathrm{~h}$. The expression level of IL- 6 could be maintained at a high level after having increased; however, the expression of ITIH4 tended to decline at $12 \mathrm{~h}$ and returned to a lower level $24 \mathrm{~h}$ after infection. With changes of mRNA expression, the expression levels of ITIH4 and IL-6 proteins in AGS cells also presented a similar response after $H$. pylori infection. These results suggestthatITIH4 is an acute phase response protein for $H$. pylori infection. IL-6 expression level reached a peak after $6 \mathrm{~h}$, and then decreased and maintained a moderate level, indicating that the elevated ITIH4 level may be mediated via IL-6. Therefore, the early rise of ITIH4 in AGS cell after H. pylori infection also suggests that it was a characteristic serum marker for the early stages of gastric cancer.

IL-6 is an inflammatory factor secreted by monocytes, endothelial cells, and various tumor cells (20). It not only plays an important role in immune response and inflammatory response, but also participates in the occurrence and development of tumors. IL-6 has two-way effects on the occurrence and development of tumors (21). For one, IL-6 can activate macrophages and prevent neutrophil apoptosis, thereby enhancing the killing effect of cancer cells; for another, IL-6 can induce vascular endothelial cell growth factor and thus contributes to tumor invasion. IL-6 can regulate the cell cycle of tumor cells through the JAK/ STAT signalling pathway and affect tumor angiogenesis (22). The results of this experiment showed that AGS cells can be induced to express IL-6 and ITIH4 at the same time after $H$. pylori infection, suggesting that IL-6 may participate in the occurrence and development of $H$. pylori-related gastric cancer. The increase in ITIH4 expression in AGS cells after $H$. pylori infection may be the result of increased IL-6 expression. In order to verify this finding, rhIL-6 was applied to induce AGS cells, and the expression of ITIH4 mRNA and protein were monitored at different IL- 6 concentrations. The results showed that the expression of ITIH4 increased at a concentration of $6.25 \mathrm{ng} / \mathrm{mL}$, and reached a plateau phase at a concentration of $50 \mathrm{ng} / \mathrm{mL}$, suggesting that there may be some restriction or feedback relationship between the expression of ITIH4 and IL-6.

ITIH4 protein is often associated with the increased expression of ITIH4 in serum under various diseases, such as rheumatoid arthritis, fatty liver infection, chronic hepatitis, and cirrhosis $(23,24)$. Persistent $H$. pylori infection can lead to sustained high levels of IL-6 expression, which in turn can positively increase the expression of ITIH4 via activating the STAT3 signalling pathway (25). Abnormal activation of STAT3 is associated with the occurrence and development of gastric cancer $(26,27)$. This also implies that the elevated levels of ITIH4 and IL-6 in peripheral serum may be relevant to assessing the risk of canceration by being able to rule out infectious disease and chronic inflammation diagnoses, especially for patients with $\mathrm{H}$. pylori infection. In short, they may function as early warning indicators of gastric cancer.

\section{Conclusions}

The detection of ITIH4 and IL-6 levels in gastric cancer cells were useful biomarkers for early gastric cancer diagnosis. The elevated ITIH4 level in gastric cancer cells is mediated by IL- 6 .

\section{Acknowledgments}

Funding: This research was supported by the Shanghai Municipal Science and Technology Commission (134119a3100).

\section{Footnote}

Data Sharing Statement: Available at http://dx.doi. org/10.21037/tcr-20-1766

Conflicts of Interest: All authors have completed the ICMJE uniform disclosure form (available at http://dx.doi. org/10.21037/tcr-20-1766). The authors have no conflicts of interest to declare.

Ethical Statement: The authors are accountable for all aspects of the work in ensuring that questions related to the accuracy or integrity of any part of the work are appropriately investigated and resolved. This study protocol was conducted in accordance with the Declaration of Helsinki (as revised in 2013) and approved by ethical committee of Shanghai General Hospital (Approval \#:2017KY210). Informed consent was not required because we only used human cancer cell line in the entire experiment.

Open Access Statement: This is an Open Access article 
distributed in accordance with the Creative Commons Attribution-NonCommercial-NoDerivs 4.0 International License (CC BY-NC-ND 4.0), which permits the noncommercial replication and distribution of the article with the strict proviso that no changes or edits are made and the original work is properly cited (including links to both the formal publication through the relevant DOI and the license). See: https://creativecommons.org/licenses/by-nc-nd/4.0/.

\section{References}

1. Bhanumathy CD, Tang Y, Monga SP, et al. ITIH4, a serine protease inhibitor regulated in interleukin-6dependent liver formation: role in liver development and regeneration. Dev Dyn 2002;223:59-69.

2. Tang Y, Kitisin K, Jogunoori W, et al. Progenitor/stem cells give rise to liver cancer due to aberrant TGF-beta and IL-6 signaling. Proc Natl Acad Sci U S A 2008;105:2445-50.

3. Soler L, Garcia N, Andres M, et al. Development and validation of an ELISA for the quantification of bovine ITIH4 in serum and milk. Vet Immunol Immunopathol 2019;217:109922.

4. Pineiro M, Alava MA, Gonzalez-Ramon N, et al. ITIH4 serum concentration increases during acutephase processes in human patients and is up-regulated by interleukin-6 in hepatocarcinoma HepG2 cells. Biochem Biophys Res Commun 1999;263:224-9.

5. Liu F, Zhang W, Yang F, et al. Interleukin-6-stimulated progranulin expression contributes to the malignancy of hepatocellular carcinoma cells by activating mTOR signaling. Sci Rep 2016;6:21260.

6. Li X, Li B, Li B, et al. ITIH4: Effective Serum Marker, Early Warning and Diagnosis, Hepatocellular Carcinoma. Pathol Oncol Res 2018;24:663-70.

7. Nakamura N, Hatano E, Iguchi K, et al. Elevated levels of circulating ITIH4 are associated with hepatocellular carcinoma with nonalcoholic fatty liver disease: from pig model to human study. BMC Cancer 2019;19:621.

8. Lee EJ, Yang SH, Kim KJ, et al. Inter-alpha Inhibitor H4 as a Potential Biomarker Predicting the Treatment Outcomes in Patients with Hepatocellular Carcinoma. Cancer Res Treat 2018;50:646-57.

9. Hathroubi S, Servetas SL, Windham I, et al. Helicobacter pylori Biofilm Formation and Its Potential Role in Pathogenesis. Microbiol Mol Biol Rev 2018;82:e0001-18.

10. Parkin DM. The global health burden of infectionassociated cancers in the year 2002. Int J Cancer 2006;118:3030-44.
11. Simondurairaj C, Krishnakumar R, Sundaram S, et al. Interleukin-6 Receptor (IL-6R) Expression in Human Gastric Carcinoma and its Clinical Significance. Cancer Invest 2019;37:293-8.

12. Chang AH, Parsonnet J. Role of bacteria in oncogenesis. Clin Microbiol Rev 2010;23:837-57.

13. Gonzalez CA, Sala N, Rokkas T. Gastric cancer: epidemiologic aspects. Helicobacter 2013;18 Suppl 1:34-8.

14. den Hoed CM, Kuipers EJ. Gastric Cancer: How Can We Reduce the Incidence of this Disease? Curr Gastroenterol Rep 2016;18:34.

15. Prinz C, Weber D. MicroRNA (miR) dysregulation during Helicobacter pylori-induced gastric inflammation and cancer development: critical importance of miR-155. Oncotarget 2020;11:894-904.

16. Taher MY, Davies DM, Maher J. The role of the interleukin (IL)-6/IL-6 receptor axis in cancer. Biochem Soc Trans 2018;46:1449-62.

17. Yousefi H, Momeny M, Ghaffari SH, et al. IL-6/IL-6R pathway is a therapeutic target in chemoresistant ovarian cancer. Tumori 2019;105:84-91.

18. Subbannayya Y, Mir SA, Renuse S, et al. Identification of differentially expressed serum proteins in gastric adenocarcinoma. J Proteomics 2015;127:80-8.

19. Huang A, Zhang M, Li T, et al. Serum Proteomic Analysis by Tandem Mass Tags (TMT) Based Quantitative Proteomics in Gastric Cancer Patients. Clin Lab 2018;64:855-66.

20. Unver N, McAllister F. IL-6 family cytokines: Key inflammatory mediators as biomarkers and potential therapeutic targets. Cytokine Growth Factor Rev 2018;41:10-7.

21. Lima PC, Moura Neto A, Tambascia MA, et al. Risk factors associated with benign and malignant thyroid nodules in autoimmune thyroid diseases. ISRN Endocrinol 2013;2013:673146.

22. Zhang Q, Xu H, Zhao J, et al. Expression and characterization of a thermostable penicillin $\mathrm{G}$ acylase from an environmental metagenomic library. Biotechnol Lett 2014;36:617-25.

23. Sira MM, Behairy BE, Abd-Elaziz AM, et al. Serum Inter-Alpha-Trypsin Inhibitor Heavy Chain 4 (ITIH4) in Children with Chronic Hepatitis C: Relation to Liver Fibrosis and Viremia. Hepat Res Treat 2014;2014:307942.

24. Noh CK, Kim SS, Kim DK, et al. Inter-alpha-trypsin inhibitor heavy chain $\mathrm{H} 4$ as a diagnostic and prognostic indicator in patients with hepatitis B virus-associated hepatocellular carcinoma. Clin Biochem 2014;47:1257-61. 
25. Jove R. Preface: STAT signaling. Oncogene 2000;19:2466-7.

26. Sansone P, Bromberg J. Targeting the interleukin-6/ $\mathrm{Jak} /$ stat pathway in human malignancies. J Clin Oncol 2012;30:1005-14.

Cite this article as: Jing D, Jin J, Mei Z, Zhu Q, Lu Y, Wang $\mathrm{X}$. Effects of Helicobacter pylori infection and interleukin 6 on the expression of ITIH4 in human gastric cancer cells. Transl Cancer Res 2020;9(8):4656-4665. doi: 10.21037/tcr-20-1766
27. Xiong H, Du W, Wang JL, et al. Constitutive activation of STAT3 is predictive of poor prognosis in human gastric cancer. J Mol Med (Berl) 2012;90:1037-46.

(English Language Editor: J. Jones and J. Gray) 